Supporting Information

\title{
The gate-modified Solution-Gated Graphene Transistors for the highly sensitive detection of lead ions
}

Bichen $\mathrm{Xiao}^{1}$, Jinhua $\mathrm{Li}^{1 *}$, Songyang Guo ${ }^{1}$, Yuexing Zhang ${ }^{2}$, Ming Peng ${ }^{1}$, Haiyang $\mathrm{Yu}^{1,3}$, Minghua Deng ${ }^{1}$, Jianying Wang ${ }^{1 *}$, Li Yu ${ }^{1}$, Xianbao Wang*

${ }^{1}$ Collaborative Innovation Center for Advanced Organic Chemical Materials Co-

constructed by the Province and Ministry, Key Laboratory for the Green Preparation and Application of Functional Materials, Ministry of Education, Hubei Key Laboratory of Polymer Materials, School of Materials Science and Engineering, Hubei University, Wuhan 430062,

China

${ }^{2}$ College of Chemistry and Chemical Engineering, Hubei University, Wuhan 430062, China

${ }^{3}$ State Key Laboratory of Advanced Technology for Materials Synthesis and Processing, Wuhan

University of Technology, Wuhan 430070, China, Biomedical Materials and Engineering Research Center of Hubei Province, Wuhan 430070, China

Corresponding authors: jinhua_li@hubu.edu.cn (J. Li), wangjy_2002@163.com (J. Wang) and wangxb68@aliyun.com (X. Wang). 


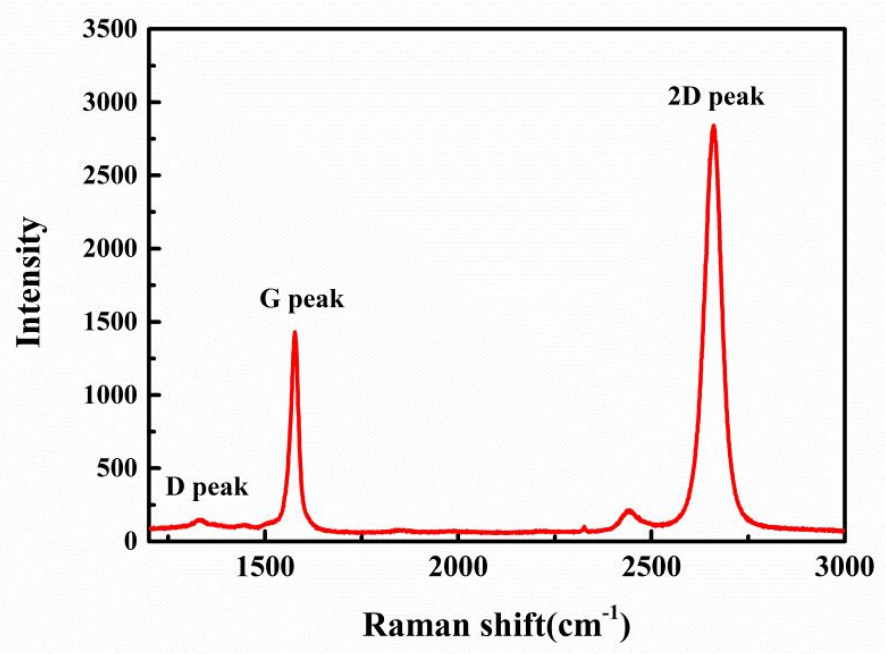

Figure S1 Raman spectrum of a single-layer CVD graphene.

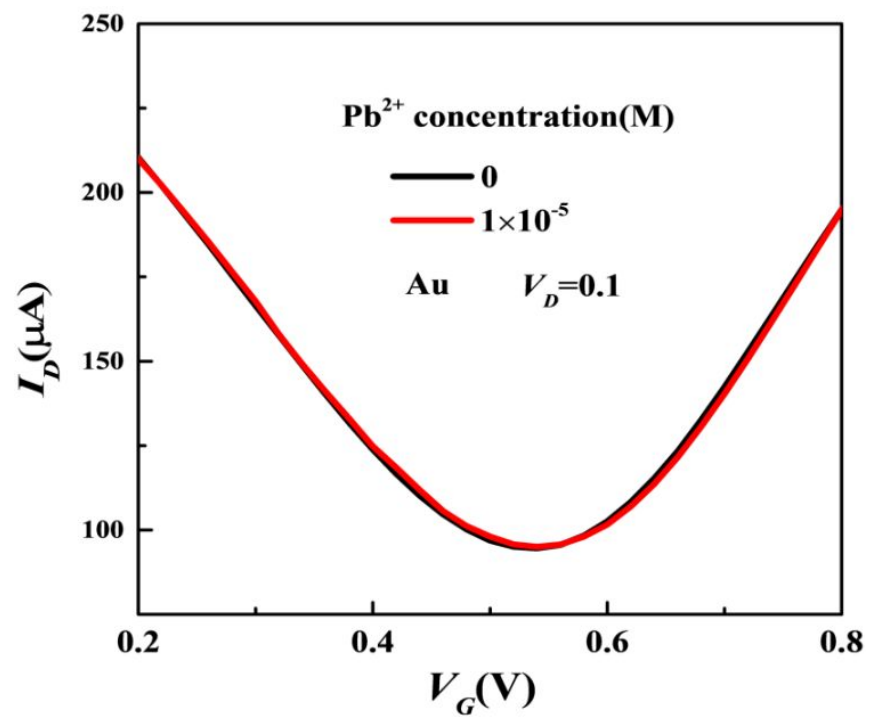

Figure S2 Transfer curves of SGGT-based sensor without the GSH modification before and after the addition of $\mathrm{Pb}^{2+}$ ions. 

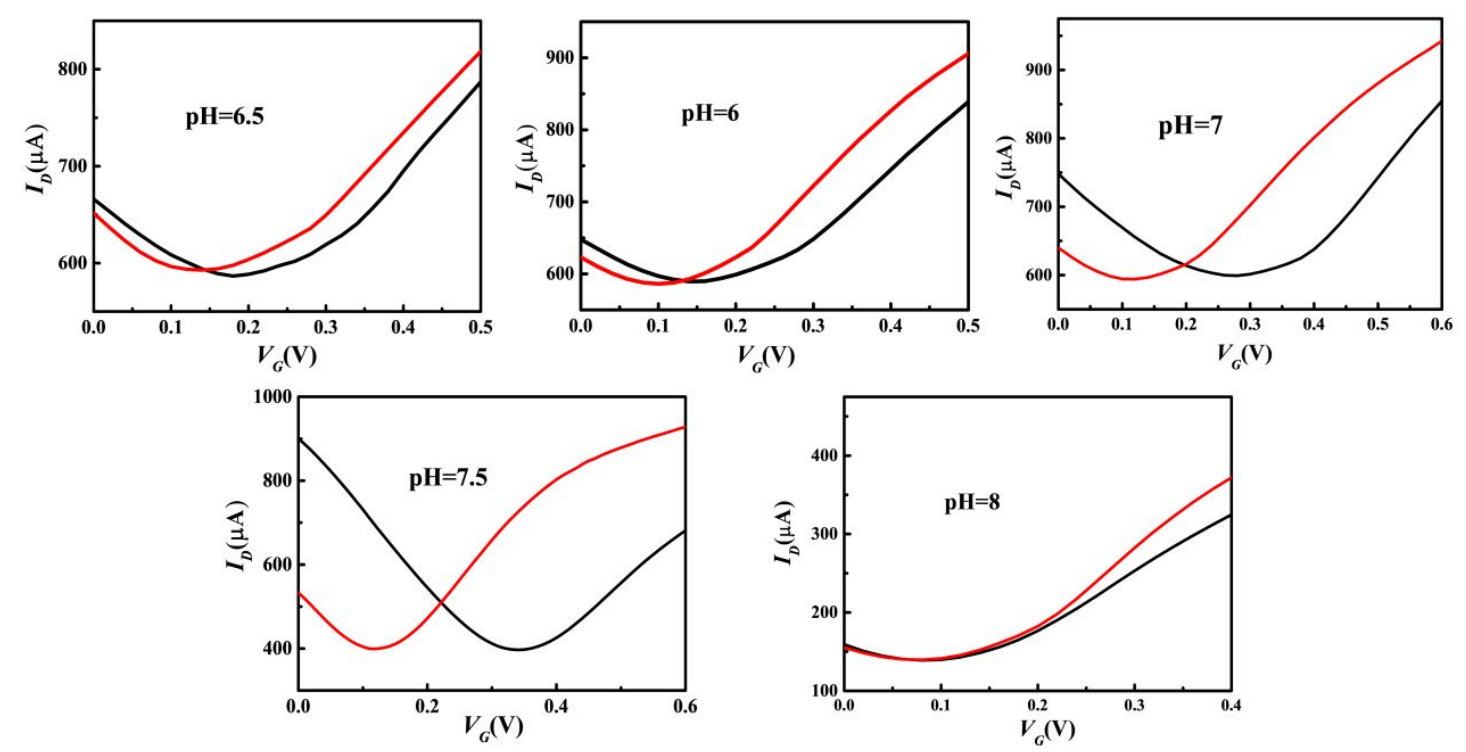

Figure S3 The transfer curves for different $\mathrm{pH}$ values (The $\mathrm{Pb}^{2+}$ ions concentration is $10^{-5} \mathrm{M}$ ).

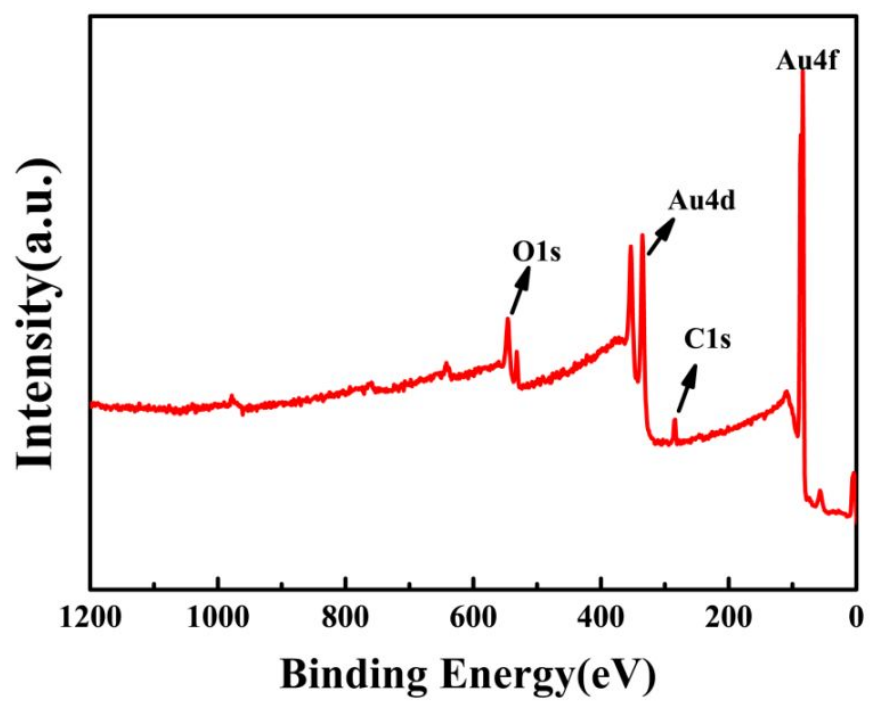

Figure S4 XPS spectrum of unmodified Au electrode. 

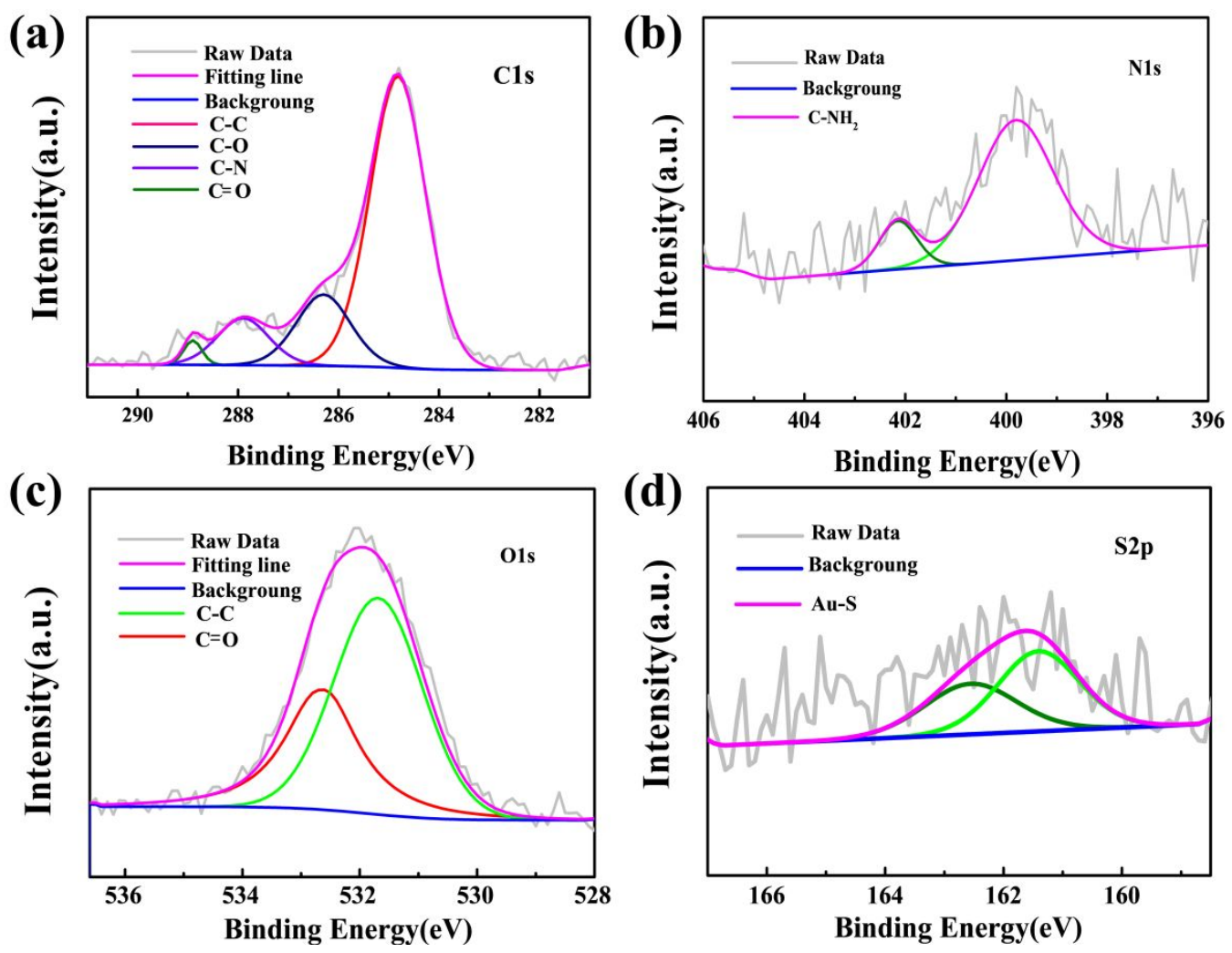

Figure S5 High-resolution C1s(a), N1s(b), O1s(c), and S2p(d) spectrum of GSH- modified Au electrode.

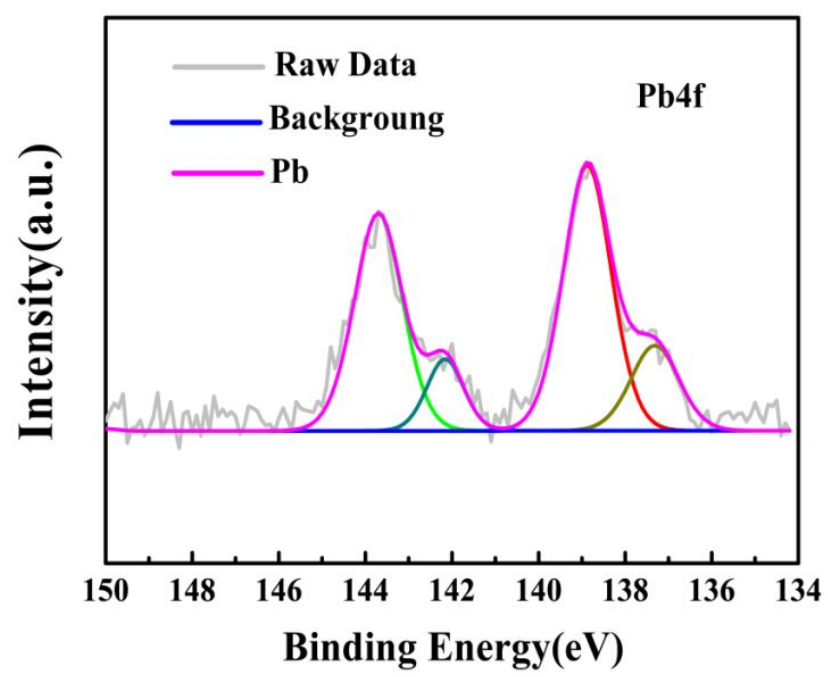

Figure S6 High-resolution $\mathrm{Pb} 4 \mathrm{f}$ spectrum of GSH- modified gold electrode after the addition of $\mathrm{Pb}^{2+}$ ion. 


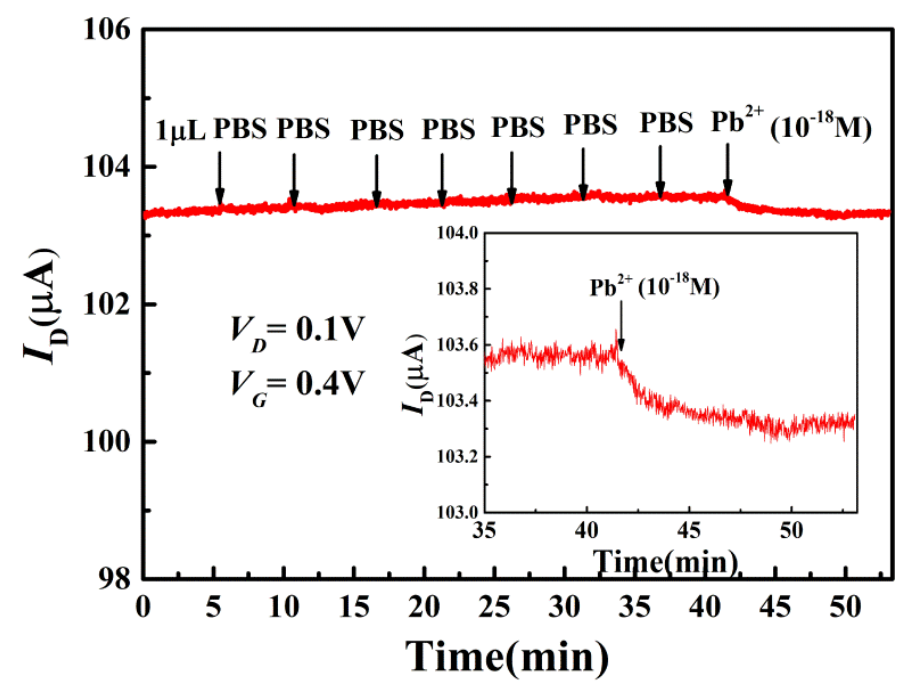

Figure S7 Time-current response of SGGT-base sensor with GSH modified on the gate electrode to 7 doses of PBS injection.

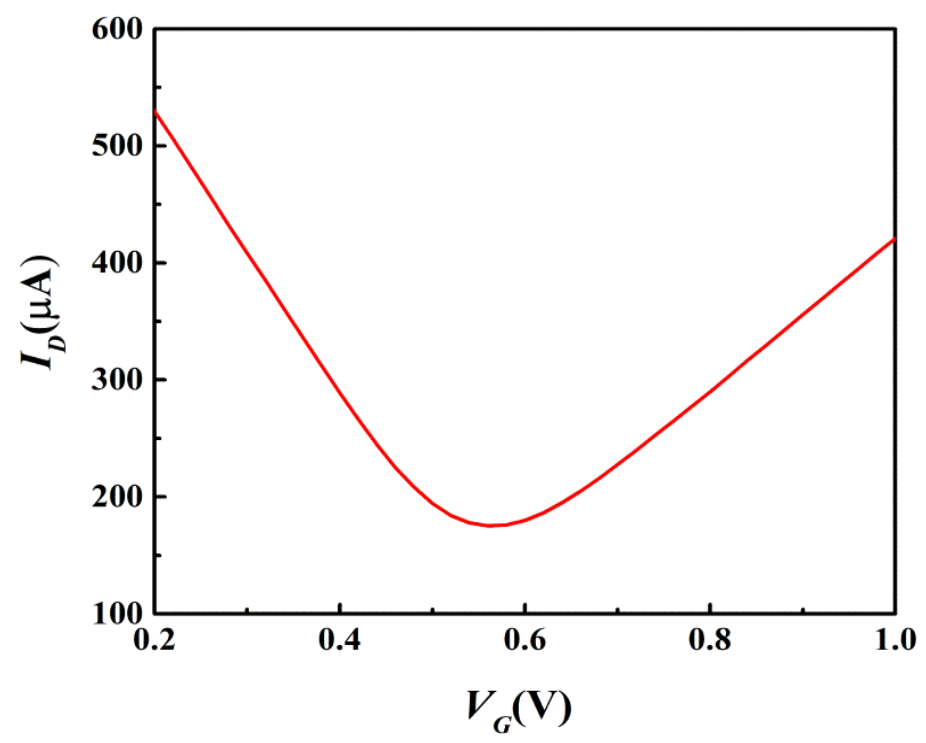

Figure S8 The transfer curve of the corresponding device in Fig.5. 


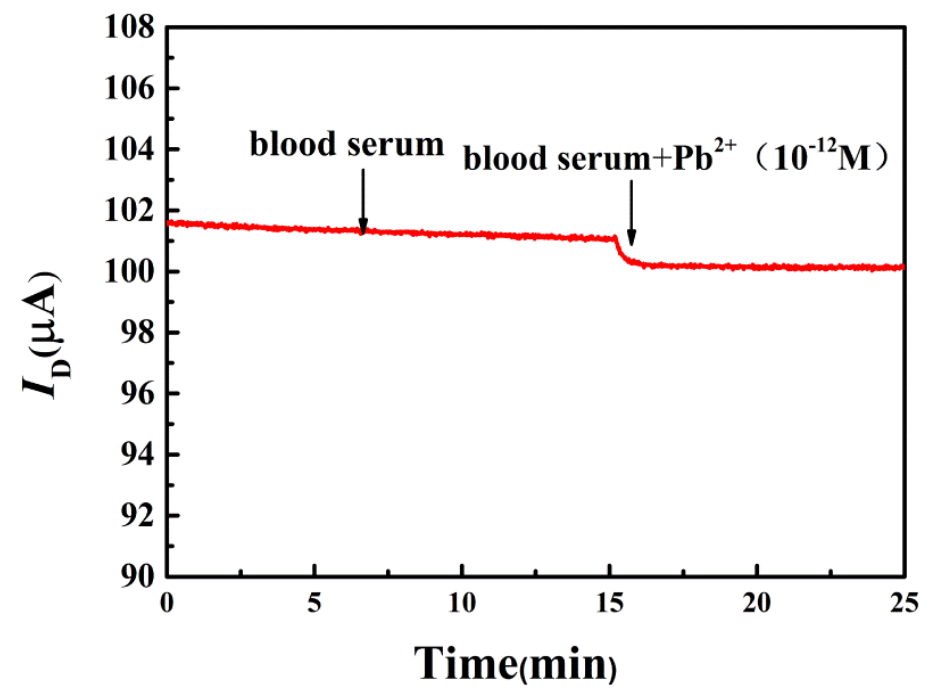

Figure S9 Time-current response of SGGT-base sensor with GSH modified on the gate electrode to the blood serum and blood serum containing lead ions $\left(\mathrm{Pb}^{2+}\right.$ concentrations is $10^{-}$ $\left.{ }^{12} \mathrm{M}\right)$

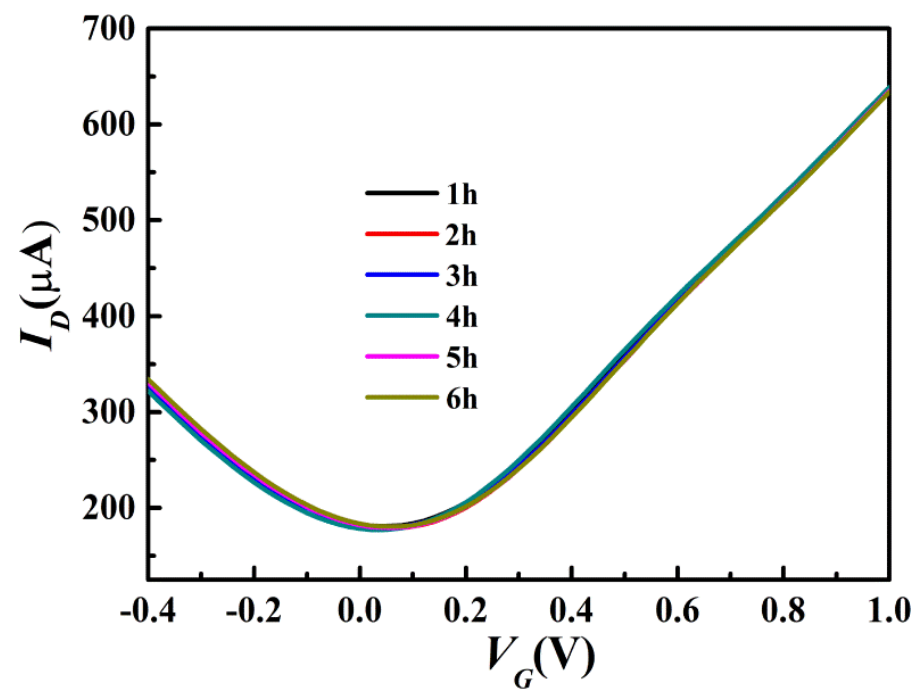

Figure S10 Time stability of GSH- modified SGGT in PBS for six hours. 


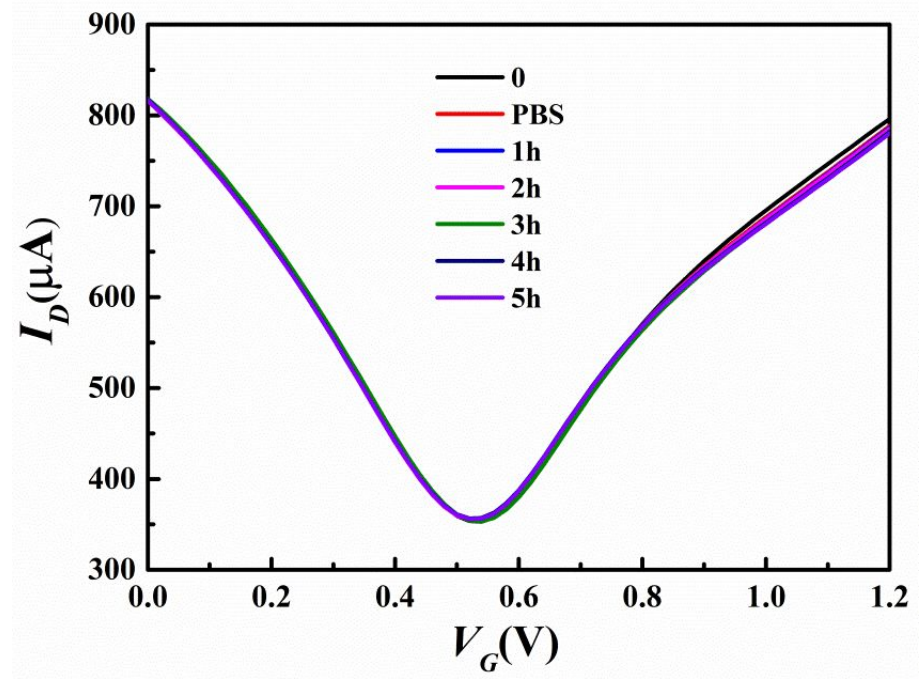

Figure S11 Time stability of GSH- modified SGGT after PBS incubation. 
Table $\mathrm{S} 1$ Recently reported various methods for detection of $\mathrm{Pb}^{2+}$ ions.

\begin{tabular}{|c|c|c|c|c|}
\hline Method & Materials & Detection limit & Linear Range & Ref. \\
\hline Fluorescent & ZnSeQDs & $0.335 \mu \mathrm{g} \mathrm{L}^{-1}$ & $1-60 \mu \mathrm{g} \mathrm{L}-1$ & 1 \\
\hline Fluorescent & CuNCs-CNQDs & $0.0031 \mathrm{mg} \mathrm{L}^{-1}$ & $0.01-2.5 \mathrm{mg} \mathrm{L}^{-1}$ & 2 \\
\hline Fluorescent & CDs-AuNCs & $26 \mathrm{nM}$ & $50-900 \mathrm{nM}$ & 3 \\
\hline Fluorescent & organic framework & $7.7 \mathrm{pM}$ & $0-11 \mathrm{nM}$ & 4 \\
\hline Fluorescent & $\mathrm{Fe}_{3} \mathrm{O}_{4^{-}}$GNPs & $5.7 \mathrm{nM}$ & $25-1400 \mathrm{nM}$ & 5 \\
\hline Fluorescent & aptasensor & $0.5 \mathrm{pM}$ & $5-70 \mathrm{pM}$ & 6 \\
\hline Fluorescent & $\mathrm{PFCeO}_{2} \mathrm{NPs}$ & $0.016 \mathrm{nM}$ & $0.05-2000 \mathrm{nM}$ & 7 \\
\hline Fluorescent & Porphyrin & $23.5 \mathrm{nM}$ & $0-600 \mathrm{nM}$ & 8 \\
\hline Electrochemical & DNAzyme & $48 \mathrm{fM}$ & $0.2 \mathrm{pM}-100 \mathrm{nM}$ & 9 \\
\hline Electrochemical & $\mathrm{Fe}_{3} \mathrm{O}_{4} @ \mathrm{SiO}_{2} @ \mathrm{IIP}$ & $0.05 \mathrm{ng} \mathrm{mL}^{-1}$ & $0.1-80 \mathrm{ng} \mathrm{mL}-1$ & 10 \\
\hline Electrochemical & DNAzyme & $33 \mathrm{fM}$ & $0.1 \mathrm{pM}-0.2 \mu \mathrm{M}$ & 11 \\
\hline Electrochemical & nanoflakes & $0.27 \mathrm{pM}$ & $0.5 \mathrm{pM}-5 \mathrm{nM}$ & 12 \\
\hline Colorimetry & GR-5 DNAzyme & $55 \mathrm{pM}$ & $0.1 \mathrm{nM}-1 \mu \mathrm{M}$ & 13 \\
\hline Colorimetry & G-quadruplex/hemin & $7.1 \mathrm{nM}$ & $0.01-10 \mu \mathrm{M}$ & 14 \\
\hline Colorimetry & RNA Cleavage & $59.39 \mathrm{pM}$ & $0 \mathrm{pM}-15 \mathrm{nM}$ & 15 \\
\hline SERS & DNAzyme & $70 \mathrm{fM}$ & $100 \mathrm{fM}-0.1 \mu \mathrm{M}$ & 16 \\
\hline SERS & DNAzyme & $0.42 \mathrm{pM}$ & $1 \mathrm{pM}-100 \mathrm{nM}$ & 17 \\
\hline Membrane & nanogels & $1 \mathrm{nM}$ & $1 \mathrm{nM}-10 \mu \mathrm{M}$ & 18 \\
\hline Interferometry & DNA Tetrahedron & $1.83 \mathrm{nM}$ & $0.1-20 \mu \mathrm{M}$ & 19 \\
\hline FET & rGO/GSH-AuNP & $10 \mathrm{nM}$ & $10 \mathrm{nM}-10 \mu \mathrm{M}$ & 20 \\
\hline
\end{tabular}




\begin{tabular}{llllr} 
apta-GFET & rGO-COOH & $1 \mathrm{pM}$ & $1-10 \mathrm{nM}$ & 21 \\
ISFET & PEDOT & $1 \mu \mathrm{g} \mathrm{L}^{-1}$ & $0-1000 \mu \mathrm{g} \mathrm{L}^{-1}$ & 22 \\
FET & PEDOT:PSS/rGO & $2.36 \mu \mathrm{g} \mathrm{L}^{-1}$ & $1-60 \mu \mathrm{g} \mathrm{L}^{-1}$ & 23 \\
& /L-Cysteine & & & 24 \\
WGTFT & SnO 2 thin-film & $0.9 \mathrm{nM}$ & $0-250 \mathrm{nM}$ & 2 \\
SGGT & glutathione & $1 \mathrm{aM}$ & $1 \mathrm{aM}-1 \mu \mathrm{M}$ & This work \\
\hline
\end{tabular}

Abbreviations: QDs, quantum dots; CuNCs-CNQDs, copper nanoclusters and nitrogen-doped carbon quantum dots; CDs, Carbon dots (CDs); AuNCs, gold nanoclusters; GNPs, gold nanoparticles; IIP, ion imprinted polymer; SERS, surfaceenhanced Raman scattering; SGGT, solution-gated graphene transistor; FET, field-effect transistor; rGO, reduced graphene oxide; apta-GFET, aptamer functionalized graphene field effect transistor; ISFET, ion selective field effect transistor; PEDOT, poly(3,4-ethylenedioxythiophene), PEDOT:PSS, poly(3,4ethylenedioxythiophene) polystyrene sulfonate; $\mathrm{SnO}_{2}$ WGTFT, water-gated $\mathrm{SnO}_{2}$ thin-film transistor, SGGT, solution-gated graphene transistor.

\section{REFERENCES:}

(1) Zhou, J.; Li, B.; Qi, A.; Shi, Y.; Chen, L. ZnSe Quantum Dot Based Ion Imprinting Technology for Fluorescence Detecting Cadmium and Lead Ions on a Three-Dimensional Rotary Paper-Based Microfluidic Chip. Sens. Actuators B 2019, 305, 127462.

(2) Li, W.; Hu, X.; Li, Q.; Shi, Y.; Zhai, X.; Xu, Y.; Li, Z.; Huang, X.; Wang, X.; Shi, J. Copper Nanoclusters@ Nitrogen-Doped Carbon Quantum Dots-Based Ratiometric Fluorescence Probe for Lead (II) Ions Detection In Porphyra. Food Chem. 2020, 320, 126623. 
(3) Lu, H.; Yu, C.; Xu, S. A Dual Reference Ion-Imprinted Ratiometric Fluorescence Probe for Simultaneous Detection of Silver (I) and Lead (II). Sens. Actuators B 2019, 288, 691-698.

(4) Venkateswarlu, S.; Reddy, A. S.; Panda, A.; Sarkar, D.; Son, Y.; Yoon, M. Reversible Fluorescence Switching of Metal-Organic Framework Nanoparticles for Use as Security Ink and Detection of $\mathrm{Pb}^{2+}$ Ions in Aqueous Media. ACS Appl. Nano Mater. 2020, 3, 3684-3692.

(5) Chen, M.; Hassan, M.; Li, H.; Chen, Q. Fluorometric Determination of Lead (II) by Using Aptamer-Functionalized Upconversion Nanoparticles and Magnetite-modified Gold Nanoparticles. Microchim. Acta 2020, 187, 1-9.

(6) Khoshbin, Z.; Housaindokht, M. R.; Izadyar, M.; Verdian, A.; Bozorgmehr, M. R. A Simple Paper-Based Aptasensor for Ultrasensitive Detection of Lead (II) Ion. Anal. Chim. Acta 2019, 1071, 70-77.

(7) Huang, Y.; Li, L.; Zhang, Y.; Zhang, L.; Ge, S.; Li, H.; Yu, J. Cerium Dioxide-Mediated Signal “On-Off” by Resonance Energy Transfer on a Lab-on-Paper Device for Ultrasensitive Detection of Lead Ions. ACS Appl. Mater. Inter. 2017, 9, 32591-32598.

(8) Peng, D.; Li, Y.; Huang, Z.; Liang, R. P.; Liu, J. Efficient DNA-Catalyzed Porphyrin Metalation for Fluorescent Ratiometric $\mathrm{Pb}^{2+}$ Detection. Anal. Chem. 2019, 91, 11403-11408.

(9) Cai, W.; Xie, S.; Zhang, J.; Tang, D.; Tang, Y. Immobilized-Free Miniaturized Electrochemical Sensing System for $\mathrm{Pb}^{2+}$ Detection Based on Dual $\mathrm{Pb}^{2+}$-DNAzyme Assistant Feedback Amplification Strategy. Biosens. Bioelectron. 2018, 117, 312-318.

(10) Dahaghin, Z.; Kilmartin, P. A.; Mousavi, H. Z. Novel Ion Imprinted Polymer Electrochemical Sensor for The Selective Detection of Lead (II). Food Chem. 2020, 303, 125374. 
(11) Zhao, J.; Jing, P.; Xue, S.; Xu, W. Dendritic Structure DNA for Specific Metal Ion Biosensor Based on Catalytic Hairpin Assembly and a Sensitive Synergistic Amplification Strategy. Biosens. Bioelectron. 2017, 87, 157-163.

(12) Wang, Y.; Shi, H.; Zhang, L.; Ge, S.; Meiling, X.; Wang, X.; Yu, J. Two-Dimensional Black Phosphorus Nanoflakes: A Coreactant-Free Electrochemiluminescence Luminophors for Selective $\mathrm{Pb}^{2+}$ Detection Based on Resonance Energy Transfer. J. Hazard. Mater. 2021, 403, 123601-123609.

(13) Chu, L. T.; Leung, H. M.; Lo, P. K.; Chen, T. Visual Detection of Lead Ions Based on Nanoparticle-Amplified Magnetophoresis and Mie Scattering. Sens. Actuators B 2020, 306, 127564.

(14) Chen, J.; Zhang, Y.; Cheng, M.; Mergny, J. L.; Ju, H. Highly Active G-quadruplex/Hemin DNAzyme for Sensitive Colorimetric Determination of Lead(II). Microchim. Acta 2019, 186, $1-8$.

(15) Huang, Z.; Chen, J.; Luo, Z.; Wang, X.; Duan, Y. Label-Free and Enzyme-Free Colorimetric Detection of $\mathrm{Pb}^{2+}$ Based on RNA Cleavage and Annealing-Accelerated Hybridization Chain Reaction. Anal. Chem. 2019, 91, 4806-4813.

(16) Tian, A.; Liu, Y.; Gao, J. Sensitive SERS Detection of Lead Ions Via DNAzyme Based Quadratic Signal Amplification. Talanta 2017, 171, 185-189.

(17) Wu, Y.; Fu, C.; Xiang, J.; Cao, Y.; Deng, Y.; Xu, R.; Zhang, H.; Shi, W. "Signal-on” SERS Sensing Platform for Highly Sensitive and Selective $\mathrm{Pb}^{2+}$ Detection Based on Catalytic Hairpin Assembly. Anal. Chim. Acta 2020, 1127, 106-113.

(18) Wang, Y.; Liu, Z.; Luo, F.; Peng, H.; Zhang, S.; Xie, R.; Ju, X.; Wang, W.; Faraj, Y.; 
Chu, L. A Novel Smart Membrane with Ion-Recognizable Nanogels as Gates on Interconnected Pores for Simple and Rapid Detection of Trace Lead (II) Ions in Water. J. Membrane Sci. 2019, $575,28-37$

(19) Wang, S.; Lu, S.; Zhao, J.; Huang, J.; Yang, X. Real-Time Study of the Interaction between G-rich DNA Oligonucleotides and Lead Ion on DNA Tetrahedron-Functionalized Sensing Platform by Dual Polarization Interferometry. ACS Appl. Mater. Inter. 2017, 9, 4156841576.

(20) Zhou, G.; Chang, J.; Cui, S.; Pu, H.; Wen, Z.; Chen, J. Real-Time, Selective Detection of $\mathrm{Pb}^{2+}$ in Water Using a Reduced Graphene Oxide/Gold Nanoparticle Field-Effect Transistor Device. ACS Appl. Mater. Inter. 2014, 6, 19235-19241.

(21) Li, F.; Wang, Z.; Jia, Y. Reduced Carboxylate Graphene Oxide based Field Effect Transistor as $\mathrm{Pb}^{2+}$ Aptamer Sensor. Micromachines-Base 2019, 10, 388.

(22) Venkatesh, S.; Li, T.; Wang, X.; Yeung, C.; Pei, K.; Sun, Q.; Wu, W.; Li, R. K.; Lam, M. H.; Chan, P. K. Dual-Gated Transistor Platform for On-Site Detection of Lead Ions at Trace Levels. Anal. Chem. 2018, 90, 7399-7405.

(23) Sayyad, P. W.; Ingle, N. N.; Al-Gahouari, T.; Mahadik, M. M.; Bodkhe, G. A.; Shirsat, S. M.; Shirsat, M. D. Sensitive and Selective Detection of $\mathrm{Cu}^{2+}$ and $\mathrm{Pb}^{2+}$ Ions Using Field Effect Transistor (FET) Based on L-Cysteine Anchored PEDOT: PSS/rGO Composite. Chem. Phys. Lett. 2020, 761, 138056.

(24) Alqahtani, Z.; Alghamdi, N.; Grell, M. Monitoring the Lead-and-Copper Rule with a Water-Gated Field Effect Transistor. J. Water Health 2020, 18, 159-171. 